\title{
Análisis de costos de financiamiento con crédito de proveedores en una empresa manufacturera mexicana
}

Analysis of financing costs with credit from suppliers in a mexican manufacturing company

Recibido: mayo22 de 2018 | Revisado: junio 26 de 2018 | Aceptado: julio 28 de 2018

JUVENCIO Roldan ${ }^{\mathrm{I}}$

MA. Karina MartíneZ

Diana GuZMÁn ${ }^{\mathrm{I}}$

\section{RESUMEN}

El presente caso es la descripción de una intervención realizada en una empresa manufacturera de gran tamaño ubicada en la Cd. De Puebla en México. Los autores describen el método utilizado para determinar los ahorros que podrían alcanzarse por pagar de contado a proveedores frecuentes y de alto volumen en materiales productivos como beneficio de obtener descuentos y así, su contrapartida por el costo de financiarse con proveedores.

Palabras clave: crédito comercial, descuento en efectivo, ahorro de compras, costos de oportunidad de capital, costos de la cadena de suministro

\begin{abstract}
The current case is the description of an intervention carried out in a large manufacturing company located in the Cd. De Puebla in Mexico. The authors describe the method used to determine the savings that could be achieved by paying in cash to frequent and high volume suppliers of productive materials as a benefit of obtaining discounts and thus, its counterpart for the cost of financing wit suppliers.
\end{abstract}

Key words: commercial credit, cash discount, shopping savings, capital opportunity costs, supply chain costs

1 Benemérita Universidad Autónoma de Puebla - México juvencioroldan@gmail.com 


\section{Introducción}

Aunque en los últimos 10 años se ha empezado a dar la importancia debida a la Cadena de Suministros buscando reducir los costos de proveeduría de materiales, las políticas de financiamiento a través de largos tiempos de pago siguen siendo prácticas comunes para la alta gerencia, particularmente en las grandes empresas manufactureras, en las que, en muchos casos, por necesidad, se financian acudiendo a plazos lo más largo posibles de pago a sus proveedores aprovechando su poder de negociación.

La globalización de las cadenas de suministro ha brindado oportunidades a los proveedores de las economías en desarrollo para convertirse en socios de la cadena de suministro de grandes empresas globales. El surgimiento de plataformas de comercio electrónico ha brindado oportunidades para que los empresarios lleguen a los clientes finales. Los proveedores de las economías en desarrollo y los pequeños y medianos proveedores de las economías desarrolladas enfrentan desafíos para acceder a los mercados de capitales. Se han creado planes de financiación innovadores, a menudo iniciados por grandes compradores solventes, para abordar esos desafíos. Entre los ejemplos se incluyen el factor inverso y el financiamiento de órdenes de compra. Los desafíos específicos que un esquema de financiamiento intenta abordar y los impactos que tienen en las decisiones operativas y el desempeño de la cadena de suministro son preguntas importantes que deben abordarse." (Dong, Kouvelis, \& Turcic, 2017).

Existen investigaciones empíricas que evidencian los impactos del tema de financiamiento con proveedores desde varias perspectivas, como: La relación del tiempo de pago con el costo de oportunidad del capital al determinar tamaños de lote con el modelo EOQ (Cantidad económica de pedido), los riesgos de no cumplimiento de las garantías de calidad por haber recibido el pago desde la entrega del producto, los riesgos de impago a acreedores bancarios por parte del cliente para cubrir sus diferentes adeudos, su valor como herramienta para ganar clientes, las variaciones de precios en los mercados de materias primas o variaciones cambiarias y su efecto en los tiempos de plazo. Incluso hay estudios sobre el cálculo del descuento a ofrecer a los compradores por pago de contado desde el punto de vista del proveedor. Sin embargo, no aparece literatura sobre los ahorros obtenidos por una empresa compradora por los descuentos obtenidos por pago de contado a su proveedor. Este artículo ofrece una evidencia al respecto.

En algunos estudios encuentran que los proveedores pequeños que financian a sus clientes obligados por estos, tienen que sacrificar inversiones en gastos de capital, y a veces de mantenimiento de sus equipos para poder soportar los largos periodos de pago de sus clientes.

Nuestras pruebas finales cuestionan si las inversiones olvidadas significaron oportunidades, perdidas (o incluso mantenimiento requerido), o si los retrasos en los pagos ayudan a disciplinar la sobreinversión de los gerentes. Consideramos el rendimiento a largo plazo de los activos en firme de la firma, luego de los cambios en la política de pago del comprador sin poder hablar directamente sobre los costos o beneficios de bienestar del gasto de inversión perdido, como una aproximación aproximada. El crecimiento en los días pagaderos por el comprador, para las empresas con limitaciones crediticias, parece preexistir a una reducción a largo plazo en la rentabilidad. Tomamos en cuenta nuestros hallazgos como muestra de que puede haber grandes costos implícitos de préstamos indirectos para vendedores con acceso limitado a créditos bancarios. (Murfin \& Njoroge, 2014) 
Conforme a la Cuenta de Flujo de Fondos de los Estados Unidos 2011, las cuentas por pagar comerciales, financiación para la compra de bienes extendidos por los proveedores a sus clientes, a partir de 2009, simbolizaron el segundo mayor pasivo en el balance agregado de negocios no financieros en los Estados Unidos. No obstante, en relación con su volumen como fuente de financiamiento corporativo, ha habido una exploración restringida sobre los efectos de las relaciones de crédito comercial en diversas actividades existentes y financieras de la empresa.

¿Por qué hay tanto financiamiento en un mercado de capitales bien desarrollado realizado por empresas no financieras? La literatura sobre crédito comercial, sugiere que la restricción financiera puede jugar un papel significativo con pruebas de que las pequeñas empresas jóvenes se basan en sus proveedores más grandes para obtener financiamiento cuando el acceso a los mercados financieros tradicionales es condicionado". (Murfin \& Njoroge, 2014)

"Si las cuentas por pagar de una empresa exceden sus cuentas por cobrar, está recibiendo financiamiento de crédito comercial. Los autores Ward y Chapman (1987), señalan este concepto al sugerir que el crédito comercial puede considerare como una fuente general de fondos. Por otra parte, para el comprador, el costo del financiamiento del crédito comercial puede ser muy alto; por ejemplo, los términos de crédito comercial tales como " $2 / 10$, neto 30 " se traduce en un costo de financiamiento de más del 37 por ciento sobre una base anual, por lo tanto, con un costo tan alto, los motivos por los que los compradores aceptan créditos comerciales y lo vendedores las ofrecen no son claras. Se han proporcionado explicaciones para esta incógnita, que se dividen en cuatro grandes categorías, tales como: fijación de precios de Nadiri(1969), condiciones macroeconómicas de Schwartz
(1974), e información de Smith (1987), y financiamiento de Emery (1984)". (Borde \& McCarty, 1988)

"Desde hace tiempo, el financiamiento basado en proveedores, conocido como crédito comercial, es un proyecto de financiamiento común existente; un proveedor ascendente, bajo este esquema, permite que el comprador intermedio postergue el pago a una fecha posterior a la fecha de la factura. Los estudios empíricos han documentado prácticas prevalentes de crédito comercial en muchas industrias, entre proveedores y compradores de diversos tamaños." (Dong , Kouvelis , \& Turcic , 2017).

Como resultado de un proceso de intervención en el área de compras de una empresa manufacturera de capital de origen local, exportadora del sector metalmecánico, se encontró que el financiamiento con proveedores puede resultar más costoso que financiarse con créditos bancarios. En dicha intervención, participó la estudiante de ingeniería industrial de la BUAP: Diana Guzmán, quien así contribuyó al desarrollo de la investigación.

La relación de la empresa compradora con sus proveedores es ya madura, es decir, de largo tiempo y existe la confianza de que el comprador pagará, tanto como de que sus proveedores cumplirían con el nivel de servicio, especificaciones de calidad y tiempos de entregas, cualquiera que sea la condición de tiempos de pago. El caso ofrece evidencia anecdótica de las respuestas de los proveedores a los cuestionamientos sobre qué porcentaje de descuento otorgarían en caso de un pago de contado. La investigación se realizó a través del siguiente proceso:

\section{1a Etapa. La selección de proveedores}

Dado que una empresa del giro y tamaño mencionado cuenta con cientos o miles de 
proveedores y adquieren una enorme cantidad de materiales, el primer paso fue seleccionar un grupo de proveedores con los que se efectuaría el sondeo relacionado con un descuento por pago de contado. El criterio lógico fue el de realizar un Pareto, 80-20, basado en un criterio de valor económico, es decir, se reunió información de todos los materiales comprados en los periodos 2015 y 2016, con los proveedores correspondientes, se calcularon montos totales, se anidaron las cifras por proveedor, se filtraron aquellos con los montos más altos, de tal forma que la suma de los montos más altos representara alrededor del 65\% de los costos de materiales, correspondiente a un porcentaje no mayor del 30\% del total de proveedores registrados. Contar con un sistema de Business Intelligence es de gran utilidad para este tipo de análisis. La Tabla 1 muestra el primer resultado de la primera etapa.

Tabla 1

Relación de montos recibidos y cancelaciones de materiales adquiridos por diferentes proveedores en los periodos 2015 y 2016

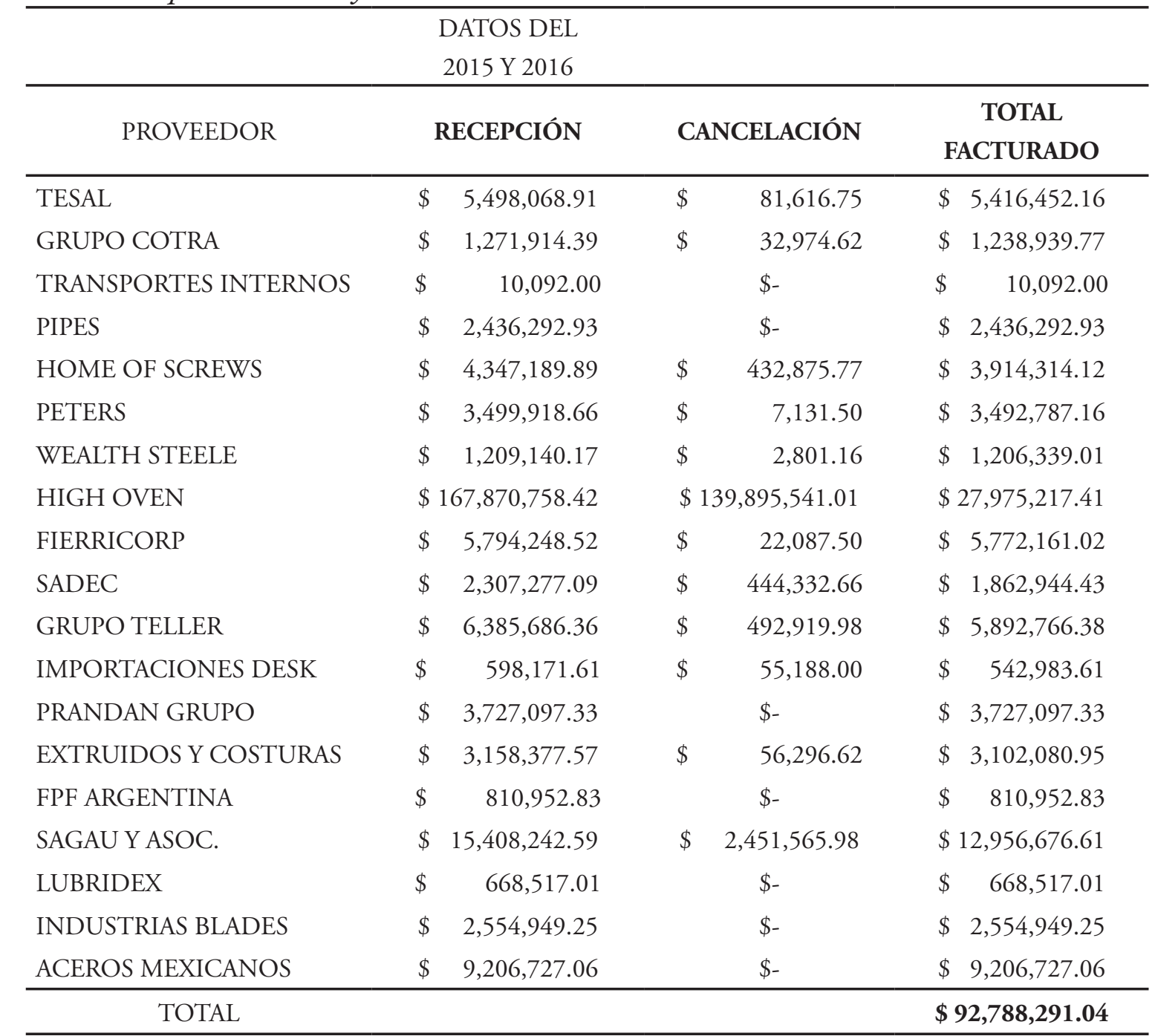

Fuente: Elaboración propia.

Cabe mencionar que en esta hay aún muchos datos irrelevantes, pero se debe aclarar que todos son reales. Previo a esta tabla, se descartaron diversos materiales que representaban un valor muy alto, pero que son suministrados por 
socios estratégicos de la compañía, con quienes una especial relación de pagos y suministros no dio cabida a negociar los descuentos.

\section{2a Etapa. El sondeo}

Si un comprador le pregunta a su prospecto de proveedor, o a su proveedor ya establecido, si le está cobrando intereses por esos 20, 30, 45, 60 ó más días de crédito, es muy probable que este responda que no, que le está cobrando los precios de lista. Por supuesto. Esa es la respuesta de la mayoría. Sí hay, sin embargo, proveedores muy fuertes, con un posicionamiento único en el mercado que plantean sus reglas muy claras y advierten explícitamente que cobrarán cierta tasa de interés, más cargos por demora, etc.

Dicha respuesta, a una pregunta incorrecta, tiene varias razones:

1. El vendedor no tiene ni la menor idea de que le está cobrando el financiamiento en los precios de lista.

2. La empresa vendedora no cobra el financiamiento porque no sabe lo que le está costando dicho financiamiento y con tal de vender están dispuestos a financiar, aunque pierdan.

3. El vendedor y su empresa saben lo que les cuesta el financiamiento, pero lo cobra tan bien en un margen de utilidad alto, que ya su negocio es financiar empresas.

Así, la pregunta correcta no es: ¡cuánto nos están cobrando por el financiamiento?, sino: ¿Qué descuento nos ofrecen por pagar de contado?

Los beneficios son notorios. Son de carácter tanto cuantitativo: Porcentajes de descuento, como cualitativo: Varios proveedores ofrecieron un descuento muy moderado, y, además, si la propuesta iba en serio, otros beneficios, tales como: Materiales en consignación, descuentos en fletes, etc. Algunos proveedores fueron más lejos al preguntar, muy interesados, si el pago sería de contado en días de pago, o contra entrega. Si el pago era contra entrega estaban dispuestos a mejorar los descuentos. Aunque adoptar dicha política en la empresa compradora implicaba llevar a cabo algunas modificaciones en sus procedimientos y sistema de programación de pagos. No llegamos a investigar si estos se justificaran.

En general, dudaban acerca del porcentaje a descontar, la mayoría dijeron una cifra tentativa, a ser consultada con sus superiores. Una de las reglas a observar durante la investigación, fue aclarar enfáticamente a los proveedores cuestionados, que solamente se estaban investigando las posibilidades y beneficios, y que no era un hecho, ni se tenía previsto un cambio de política. Cabe mencionar que a todos los proveedores les entusiasmó la propuesta y sugirieron que deberían llevarlo a la práctica. Estos comentarios nos hacen pensar que el cambiar la política de pagos y pagar de contado, no solamente permite grandes ahorros, sino que también fortalece la posición negociadora de la empresa compradora y su imagen ante todos sus proveedores.

\section{¿Por qué el financiamiento por provee- dores es el más costoso?}

Para responder a esta pregunta, es necesario destacar que los bancos son instituciones cuya actividad primordial es la de colectar y prestar dinero, por lo tanto, 
cuentan con una infraestructura especializada en investigación de créditos y cobranza. Este costo fijo de infraestructura jurídica proviene de servir a miles de clientes. Efectivamente, cuentan con economías de escala hablando de análisis y cobranza de créditos. Es su negocio. Y aunque en las economías en desarrollo es más costoso que en otros lugares del mundo, como los países más desarrollados, no deja de ser una fuente primaria de financiamiento.

Al decir fuente primaria, se debe considerar al capital propio, o de los socios, como otra fuente primaria. Es decir: ¿para qué endeudarse si se tiene el capital?. ¿Para qué pagar los altísimos intereses cuando se cuenta con dicho capital?, ya sea reunido por ahorros propia o de conocidos. Pero muchos no consideran que dicho capital también tiene un costo, que sería más alto que el de la deuda con bancos. ¿Porqué? Un socio capitalista espera al menos los intereses de una inversión libre de riesgo, como la de los certificados de tesorería (CETES), que, aunque poco, nunca dejará de pagarle sus rendimientos respectivos, más una prima de riesgo asumido por invertirlo en un negocio con una alta incertidumbre. ¿Deja más un negocio propio que invertir en CETES? Seguro, por supuesto, tal vez cientos de veces más. Pero en el negocio se está expuesto a perder todo también. $\mathrm{O}$ bien a que el administrador del negocio desaparezca o se declare en no pago, y en nuestro país, un juicio para recuperar el dinero puede llevarse años. Es por eso que el socio espera al menos una tasa similar a la bancaria más el correspondiente premio por riesgo asumido, el cual dependerá de la aversión al riesgo del inversionista y del grado de riesgo del negocio.
En el ámbito de pequeñas y medianas empresas determinan tales primas de riesgo en forma arbitraria, con rangos que pueden oscilar de un 10 a un $25 \%$, más tasa la tasa de descuento que paga la prima libre de riesgo del CETE, supongamos $7 \%$ anual. $Y$ eso hace que se le tenga que pagar al socio hasta un 32\%. Si el socio, que asume también el riesgo de perder, prevé que no obtendrá al menos ese rendimiento, mejor no entrará al negocio, o bien se saldrá a la primera oportunidad. Por otra parte, el banco jamás pierde. Se asegura siempre de cobrar, con un costo fijo, cobrado en la tasa de interés establecida, que puede oscilar entre un 11 y un 17\% anual, o bien establecido en el contrato como gastos extras de cobranza.

Desde esta perspectiva, el proveedor es una fuente secundaria de financiamiento, ya que se financia:

- A través del banco (en el mejor de los casos)

- A través de socios (más caro que el banco por lo explicado anteriormente)

- A través de sus proveedores (los que se pueden financiar por banco, socios o sus proveedores)

Si el proveedor no está consciente de que su financiamiento le cuesta, es porque no lo contabiliza. Pero todos saben hacer cuentas, así que le van a cobrar a sus clientes ese costo, más su respectiva prima de riesgo y gastos de cobranza. $\mathrm{Y}$ así se construye una costosa cadena de financiamientos y su efecto látigo. Cuando se les ha cuestionado a algunos compradores sobre si están conscientes de que sus proveedores les cobran caro el financiamiento, algunos responden cínicamente que sí, 
y que es muy caro, pero que su compañía se lo cobra de todas formas a los clientes. A pesar de todo, se debe reconocer que en México hay empresas muy conscientes de la importancia de su liquidez, de la rotación de sus flujos de efectivo, y que estimulan el pago de contado.

Una empresa global instalada en México que paga de contado a sus proveedores es Procter\&Gamble, y ESPINTEX, de un propietario único. Así también, se ha conversado con empresas proveedoras a las que no les interesa promover el pago de contado, pues admiten que cobran muy bien el pago a plazo, y su negocio ya radica en esa ganancia por financiamiento.

\section{Etapa 3. El análisis previo}

En cuestión de unos días, se logró tener la respuesta de todos los proveedores de nuestra lista 80-20. Dando lugar a una tabla como la siguiente:

Tabla 2

Porcentaje de descuento que cada proveedor otorga.

\begin{tabular}{lc}
\hline \multicolumn{1}{c}{ PROVEEDOR } & $\begin{array}{c}\% \text { de } \\
\text { Descuento }\end{array}$ \\
\hline TESAL & $3.00 \%$ \\
GRUPO COTRA & $2.00 \%$ \\
TRANSPORTES INTERNOS & $3.00 \%$ \\
PIPES & $0.00 \%$ \\
HOME OF SCREWS & - \\
PETERS & $0.00 \%$ \\
WEALTH STEELE & $1.25 \%$ \\
HIGH OVEN & $1.50 \%$ \\
FIERRICORP & $0.00 \%$ \\
SADEC & - \\
GRUPO TELLER & $1.00 \%$ \\
IMPORTACIONES DESK & $0.00 \%$ \\
PRANDAN GRUPO & $1.00 \%$ \\
EXTRUIDOS Y COSTURAS & $0.00 \%$ \\
FPF ARGENTINA & $3.00 \%$ \\
SAGAU Y ASOC. & $1.50 \%$ \\
LUBRIDEX & $4.00 \%$ \\
INDUSTRIAS BLADES & $3.00 \%$ \\
ACEROS MEXICANOS & $0.75 \%$ \\
\hline FUI
\end{tabular}

Fuente: Elaboración propia

Como se puede observar, algunos no ofrecieron descuento alguno, tal vez por las razones que se han comentado previamente. En otros casos, parecen ser muy pequeños. $0.75 \%$, 1.0 y máximos de $4 \%$.
Pero no perdamos de vista que estas fueron propuestas casi automáticas, sin ser consultadas y sin negociaciones más avanzadas. Por lo tanto, podrían mejorar tanto en lo cuantitativo como en lo cualitativo. 


\section{Etapa 4. El análisis a fondo}

A primera vista, además, puede parecer frustrante obtener descuentos máximos de $4 \%$, cuando simplemente los CETES a 28 días ofrecen tasas de descuento de aproximadamente el 7\% anual. Así que puede parecer que en el caso de la empresa en la que se hizo el análisis, que dispone del capital en efectivo, pero que prefiere tenerlo invertido en CETES, el costo de oportunidad superaría a los ahorros por los descuentos. Sin embargo, al aplicar los descuentos a los montos y frecuencia de compras a lo largo del ańo, las cifras se ven muy diferentes como se muestran en la Tabla 3.

Tabla 3

Ahorro anual por proveedor por descuento

\begin{tabular}{|c|c|c|c|c|c|}
\hline PROVEEDOR & $\begin{array}{c}\% \text { Ofrecido de } \\
\text { Descuento }\end{array}$ & & $\begin{array}{l}\text { TAL ANUAL } \\
\text { ICTURADO }\end{array}$ & & Ahorro anual \\
\hline TESAL & $3,00 \%$ & $\$$ & $5,416,452.16$ & $\$$ & $162,493.56$ \\
\hline GRUPO COTRA & $2,00 \%$ & $\$$ & $1,238,939.77$ & $\$$ & $24,778.80$ \\
\hline $\begin{array}{l}\text { TRANSPORTES } \\
\text { INTERNOS }\end{array}$ & $3,00 \%$ & $\$$ & $10,092.00$ & $\$$ & 302.76 \\
\hline PIPES & $0,00 \%$ & & & $\$$ & - \\
\hline HOME OF SCREWS & - & & & $\$$ & - \\
\hline PETERS & $0,00 \%$ & & & $\$$ & - \\
\hline WEALTH STEELE & $1,25 \%$ & $\$$ & $1,206,339.01$ & $\$$ & $15,079.24$ \\
\hline HIGH OVEN & $1,50 \%$ & $\$$ & $27,975,217.41$ & $\$$ & $419,628.26$ \\
\hline FIERRICORP & $0,00 \%$ & & & $\$$ & - \\
\hline SADEC & - & & & $\$$ & - \\
\hline GRUPO TELLER & $1,00 \%$ & $\$$ & $5,892,766.38$ & $\$$ & $58,927.66$ \\
\hline $\begin{array}{l}\text { IMPORTACIONES } \\
\text { DESK }\end{array}$ & $0,00 \%$ & & & $\$$ & - \\
\hline PRANDAN GRUPO & $1,00 \%$ & $\$$ & $3,727,097.33$ & $\$$ & $37,270.97$ \\
\hline $\begin{array}{l}\text { EXTRUIDOS Y } \\
\text { COSTURAS }\end{array}$ & $0,00 \%$ & & & $\$$ & - \\
\hline FPF ARGENTINA & $3,00 \%$ & $\$$ & $810,952.83$ & $\$$ & $24,328.58$ \\
\hline SAGAU Y ASOC. & $1,50 \%$ & $\$$ & $12,956,676.61$ & $\$$ & $194,350.15$ \\
\hline LUBRIDEX & $4,00 \%$ & $\$$ & $668,517.01$ & $\$$ & $26,740.68$ \\
\hline INDUSTRIAS BLADES & $3,00 \%$ & $\$$ & $2,554,949.25$ & $\$$ & $76,648.48$ \\
\hline ACEROS MEXICANOS & $0,75 \%$ & $\$$ & $9,206,727.06$ & $\$$ & $69,050.45$ \\
\hline TOTAL & & $\$$ & $71,664,726.82$ & & 109,599.59 \\
\hline
\end{tabular}

Fuente: Elaboración propia

Como se puede apreciar, existe un ahorro anual de \$1, 109,599.60 m.n. Aunque aún falta considerar el costo de oportunidad. En el caso de la empresa en la que se llevó a cabo la investigación, esta cuenta con la liquidez como para hacer los pagos de contado, no obstante, $y$ en forma conservadora lo tienen inverti- 
do en CETES a 28 días, inversión muy segura y por lo tanto con rendimientos muy bajos.

El costo de oportunidad se vuelve bastante relativo, porque depende de la rotación de la inversión en inventarios. Por ejemplo, si esta rotación de inversión es de 12 veces al año, para pagar de con- tado, la empresa necesita un capital de aproximadamente 6 millones de pesos, que le generan unos $\$ 420,000$ anuales basados en una tasa anual aproximada del 7\%. Al restar este costo de oportunidad al casi millón y 110 mil pesos, el beneficio sigue siendo de unos $\$ 690,000$ pesos al año (Tabla 4).

Tabla 4

Diferencia anual por costo de oportunidad financiero

\begin{tabular}{|c|c|c|c|c|c|c|}
\hline $\begin{array}{l}\text { INVERSIÓN EN } \\
\text { INVENTARIOS }\end{array}$ & \multicolumn{2}{|c|}{$\$ 6,000,000.00$} & \multicolumn{2}{|c|}{$\$ 4,000,000.00$} & \multicolumn{2}{|c|}{$\$ 3,000,000.00$} \\
\hline ROTACIÓN & \multicolumn{2}{|c|}{$\begin{array}{l}12 \text { al año ( } 1 \text { mes de } \\
\text { inventarios) }\end{array}$} & \multicolumn{2}{|c|}{$\begin{array}{l}18 \text { al año (3 semanas } \\
\text { de inventarios) }\end{array}$} & \multicolumn{2}{|c|}{$\begin{array}{l}24 \text { al año (2 semanas } \\
\text { de inventarios) }\end{array}$} \\
\hline Ahorro anual por descuentos & $\$$ & $1,110,000.00$ & $\$$ & $1,110,000.00$ & $\$$ & $1,110,000.00$ \\
\hline $\begin{array}{l}\text { Beneficio anual obtenido en } \\
\text { CETES a } 7 \%\end{array}$ & & $420,000.00$ & $\$$ & $280,000.00$ & $\$$ & $210,000.00$ \\
\hline $\begin{array}{l}\text { Diferencia anual a favor de la } \\
\text { empresa }\end{array}$ & & $690,000.00$ & $\$$ & $830,000.00$ & $\$$ & $900,000.00$ \\
\hline
\end{tabular}

Fuente: Elaboración propia.

Así, más que nunca serían útiles las estrategias de reducción de inventarios y una negociación efectiva con los proveedores. Por no mencionar los beneficios fiscales y de aprovechamiento de espacios. En términos de la TIR que resulta de cada estrategia, revisen la Tabla 5, en la que se comparan los retornos obtenidos por invertir en CETES, contra los obtenidos por utilizar el efectivo para pagar de contado, considerando tres niveles de rotación de materiales.

Tabla 5

Comparativo de beneficios por rotación de materiales

\begin{tabular}{lcccc}
\hline CETES 1 AÑO & $\begin{array}{c}\text { Pago contado } \\
\text { Rotación 12 } \\
\text { anual }\end{array}$ & $\begin{array}{c}\text { Pago contado } \\
\text { Rotación 18 } \\
\text { anual }\end{array}$ & $\begin{array}{c}\text { Pago contado } \\
\text { Rotación 24 anual }\end{array}$ \\
\hline INVERSIÓN & $\$ 6.000 .000,00$ & $-\$ 6,000,000$ & $-\$ 4,000,000$ & $-\$ 3,000,000$ \\
MONTO FINAL & $\$ 6,420,000.00$ & $\$ 7,110,000$ & $\$ 5,110,000$ & $\$ 4,110,000$ \\
TIR & $\mathbf{7 \%}$ & $\mathbf{1 8} \%$ & $\mathbf{2 8 \%}$ & $\mathbf{3 7 \%}$ \\
\hline
\end{tabular}

Fuente: Elaboración propia

Esto es mucho más revelador. Sin embargo, no todas las empresas pueden darse el lujo de pagar de contado en lugar de colocar su dinero en una inversión libre de riesgo a un costo de oportunidad financiero tan bajo. ¿Qué tanto aplica esto 
en el caso de pequeñas empresas que no disponen de este capital y que tendrían que pedir un préstamo bancario para financiar estas operaciones?

Suponiendo que el banco preste seis, cuatro o tres millones de pesos, factible para una empresa operando y que demuestra flujos de efectivo a una tasa anual estimada del 17\% como lo marca el banco de México, también dependería de la rotación de inventarios, aun así, hay beneficios que permitirían cubrir el costo financiero y en pocos años pagar su deuda y tener su propio capital de trabajo. La Tabla 6 así lo evidencia.

Tabla 6

Flujos netos de efectivo a una tasa anual estimada del 17\%.

\begin{tabular}{|c|c|c|c|c|c|c|}
\hline $\begin{array}{l}\text { INVERSIÓN EN } \\
\text { INVENTARIOS }\end{array}$ & \multicolumn{2}{|c|}{$\$ 6,000,000.00$} & \multicolumn{2}{|c|}{$\$ 4,000,000.00$} & \multicolumn{2}{|c|}{$\$ 3,000,000.00$} \\
\hline Rotación & \multicolumn{2}{|c|}{$\begin{array}{l}12 \text { al año ( } 1 \text { mes de } \\
\text { inventarios) }\end{array}$} & \multicolumn{2}{|c|}{$\begin{array}{l}18 \text { al año ( } 3 \text { semanas } \\
\text { de inventarios) }\end{array}$} & \multicolumn{2}{|c|}{$\begin{array}{l}24 \text { al año ( } 2 \text { semanas } \\
\text { de inventarios) }\end{array}$} \\
\hline $\begin{array}{l}\text { Ahorro anual por } \\
\text { descuentos }\end{array}$ & $\$$ & $1,110,000.00$ & $\$$ & $1,110,000.00$ & $\$$ & $1,110,000.00$ \\
\hline $\begin{array}{l}\text { Costo de deuda bancaria } \\
\text { a tasa anual de } 17 \%\end{array}$ & $\$$ & $1,020,000.00$ & $\$$ & $680,000.00$ & $\$$ & $510,000.00$ \\
\hline Diferencia anual a favor & $\$$ & $90,000.00$ & $\$$ & $430,000.00$ & $\$$ & $600,000.00$ \\
\hline
\end{tabular}

Fuente: Elaboración propia.

Como los lectores pueden reconocer, es más económico financiarse con el banco que con los proveedores. En este caso real, se obtienen ahorros importantes, sin considerar aún la posibilidad de los ahorros que se pueden alcanzar en una negociación más agresiva. En algunos países de la Unión Europea tienen ubicado este problema y sus respectivos gobiernos han tomado medidas severas al respecto.

En España, a partir del 2005, entró en vigor una ley que castiga a los deudores comerciales que demoren más de 30 días en cubrir sus compromisos monetarios, con una tasa especial y una compensación por los gastos de cobranza. El autor asume intuitivamente que, si toda la cadena de suministros decide financiarse con plazos de pago, entonces se genera un efecto látigo, cuyos costos acumulados de financiamiento se reflejan en precios más altos a los consumidores finales de una región comercial específica.

"En la actualidad, para determinar el período óptimo de crédito comercial se está prestando cada vez más atención por parte de los investigadores; hay dos puntos de vista principales de investigación, uno es la perspectiva del proveedor de crédito comercial, y otro es la perspectiva del juego. Desde la perspectiva del proveedor de crédito comercial, por ejemplo, Lou y Wang (2012) extendieron el modelo de Teng y Lou (2012) para establecer un modelo EOQ para derivar el período óptimo de crédito comercial y el tamaño del lote simultáneamente. Pero, en su modelo, no tenían que ver con el beneficio del minorista 
y una oportunidad de capital adicional les costaba a los proveedores la carga de ofrecer crédito comercial."(Chengfeng \& Qiuhong, 2016).

Recientemente, Teng et al. (2014) extendieron el modelo de Lou y Wang (2012) para considerar el fenómeno de la curva de aprendizaje y la pérdida de oportunidad de capital durante el período de demora en el pago.(Chengfeng \& Qiuhong, 2016). “A partir de 2009, las cuentas comerciales por pagar -financiación para la compra de bienes extendidos por los proveedores a sus clientesrepresentaron el segundo mayor pasivo en el balance agregado de las empresas no financieras en los Estados Unidos (Cuenta de flujo de fondos de los Estados Unidos 2011)." (Murfin \& Njoroge, 2014).

\section{Conclusiones}

La investigación realizada en la intervención en la empresa demostró cuantitativa y cualitativamente que la política de financiar materiales con crédito de proveedores tiene un costo superior al del financiamiento bancario. Al entrevistar por separado a otros directores, siempre argumentan que financiarse con proveedores mejora sus indicadores de liquidez, pero no consideran que el costo de tal liquidez es superior al del financiamento bancario para tales efectos.

Quedan oportunidades de investigación considerando que hay casos de proveedores cuyos corporativos están en los Estados Unidos, Europa o Japón, en donde las tasas de interés son mucho más bajas, con los correspondientes costos inferiores de fondeo, a pesar de que cobren alguna prima de riesgo a sus clientes.

\section{Referencias}

Bigus, J. (n.d.). Benefits and costs of having bank and trade credit simultaneously. University of Osnabrück, Department of Economics, 19.

Borde, S., \& McCarty, D. (1998). Determining the cash discount in the firm's credit policy: an evaluation. Journal of Financial and Strategic Decisions, 9.

Chengfeng, W., \& Qiuhong, Z. (2016). Two retailer-supplier supply chain. 22.

Dong , L., Kouvelis , P., \& Turcic , D. (2017). Supply Chain Finance. Te- chnology, Information and Operations Management, 19.

Fabbri, D. (2013). Bargaining Power and Trade Credit. Cass Business School, 36.

Lou KR, Wang WC (2012) Optimal trade credit and order quantity when trade credit impacts on both demand rate and default risk. J Oper Res Soc 64.

Murfin, J., \& Njoroge, K. (2014). The Implicit Costs of Trade Credit Borrowing by Large Firms. 51. 
Sattar, Z. (2000). Bangladesh Suppliers' Credit as External Finance Challenges for Fiscal and External Debt Management. 32.
Teng JT, Lou KR (2012) Seller's optimal credit period and replenishment time in a supply chain with upstream and down- stream trade credits. J Glob Optim 53. 